\title{
Occlusion of Internal Carotid Artery and Formation of Anterior Communicating Artery Aneurysm in Cervicocephalic Fibromuscular Dysplasia
}

\author{
-Follow-up Case Report-
}

\author{
Youichi ITOYAMA, Shodo FujIOKA, Shuichi TAKAKI, Motohiro MORIOKA, \\ Takuichiro HIDE, and Yukitaka USHIO* \\ Section of Neurosurgery, Saiseikai Kumamoto Hospital, Kumamoto; \\ ${ }^{*}$ Department of Neurosurgery, Kumamoto University Medical School, Kumamoto
}

\begin{abstract}
A 44-year-old female with cervicocephalic fibromuscular dysplasia (FMD) suffered a subarachnoid hemorrhage 10 years after presenting with left impaired extraocular movement. Carotid and vertebral angiography had shown string-of-beads sign in the left external carotid artery and fusiform aneurysms in the left carotid cavernous portion and in the left vertebral artery. On admission, the left carotid angiogram showed complete occlusion of the left internal carotid artery and the right carotid angiogram showed a new anterior communicating artery aneurysm. The territory of the left middle cerebral artery was supplied from the right internal carotid artery via the anterior communicating artery and from the basilar artery via the posterior communicating artery. Occlusion of the main arteries is rare in the clinical course of FMD. We suggest that hemodynamic stress due to occlusion of the internal carotid artery contributed to formation of the anterior communicating artery aneurysm, possibly associated with intrinsic changes of the arterial wall induced by FMD.
\end{abstract}

Key words: fibromuscular dysplasia, aneurysm formation, internal carotid artery occlusion, subarachnoid hemorrhage

\section{Introduction}

Cervicocephalic fibromuscular dysplasia (FMD) is more common in Western countries where 300 cases have occurred, ${ }^{12)}$ compared to about 20 in Japan. ${ }^{10}$ This epidemiological disparity, and its predominance among middle-aged females, are well known. Cervicocephalic FMD is accompanied by an intracranial aneurysm in $21-51.4 \%$ of cases, suggesting that FMD may cause predisposition to the formation of intracranial aneurysm..$^{7,12,13,18,19)}$ George et al. ${ }^{6)}$ found that cervicocephalic FMD carries a poor prognosis due to the high rate of associated vasospasm following rupture of aneurysms.

We describe a 44-year-old Japanese female, who had been diagnosed as having cervicocephalic FMD and intracranial fusiform aneurysms 10 years ear-

Received October 1, 1993; Accepted January 28, 1994 lier, ${ }^{10)}$ with subarachnoid hemorrhage (SAH) due to rupture of a newly formed anterior communicating artery aneurysm and complete occlusion of the left internal carotid artery.

\section{Case Report}

This 44-year-old female had previously developed impairment of left extraocular movement and double vision in January, 1983. Angiography then revealed string-of-beads sign in the left external carotid artery, a giant fusiform aneurysm in the left carotid cavernous portion, and another fusiform aneurysm in the left vertebral artery. The left internal carotid artery was patent, and no aneurysm was present in the anterior communicating artery (Fig. 1). She was treated conservatively.

On July 27, 1992, she experienced a sudden severe headache, and was admitted to our hospital. Neurological examination found she was alert 

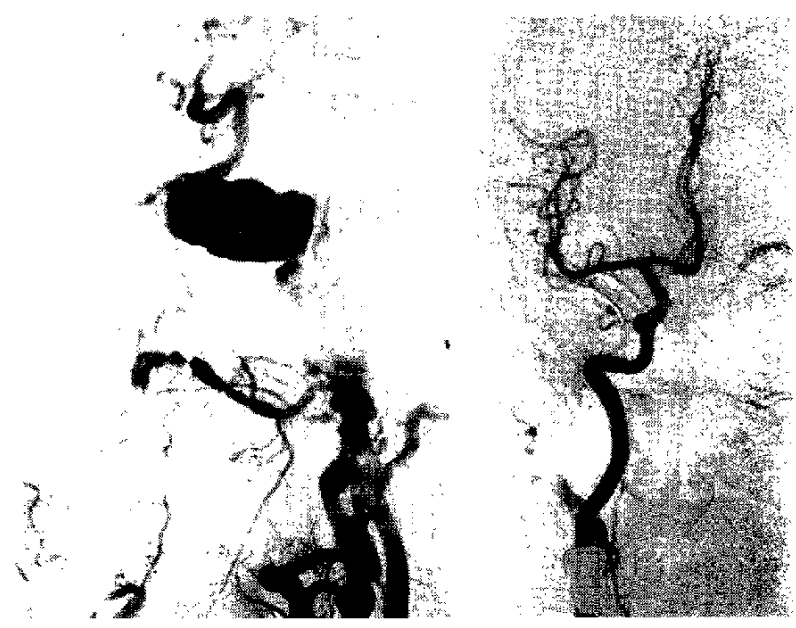

Fig. 1 left: Left carotid angiogram, lateral view, at first examination showing string-of-beads sign in the left external carotid artery and a giant fusiform aneurysm in the left cavernous sinus. The left internal carotid artery is patent. right: Right carotid angiogram, anteroposterior view, at first examination showing no aneurysm in the anterior communicating artery.

without paresis, but her headache and neck stiffness were severe (Hunt and Kosnik grade 2). Left abducens nerve paresis with the absence of light reflex, resulting from the giant fusiform aneurysm in the left cavernous sinus, were also present. These symptoms had persisted for the last 10 years. Com-

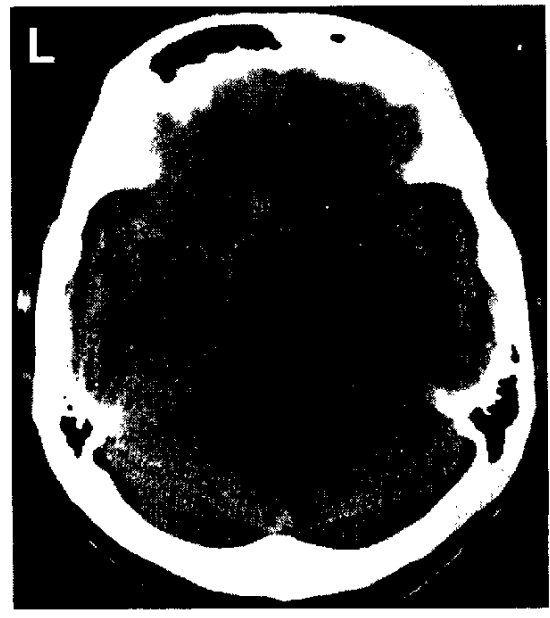

Fig. 2 CT scan on admission showing SAH.

puted tomography (CT) on this admission showed SAH, Fisher group 2 (Fig. 2). A left carotid angiogram revealed string-of-beads sign in the left external carotid artery and complete occlusion of the left internal carotid artery at a point near the bifurcation of the common carotid artery (Fig. 3 left). A right carotid angiogram showed a saccular aneurysm arising from the anterior communicating artery which had not been present 10 years earlier (Fig. 3 center). This aneurysm was thought to be the origin of the SAH. A left vertebral angiogram revealed the fusiform aneurysm in the left vertebral artery which had been noted 10 years earlier (Fig. 3
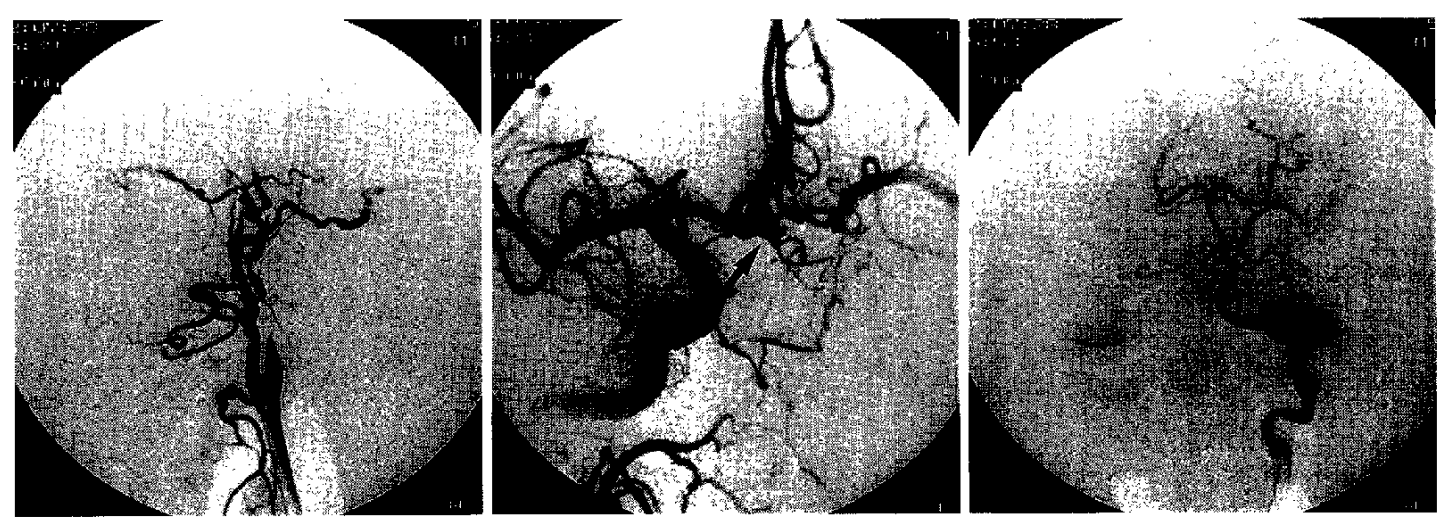

Fig. 3 left: Left carotid angiogram, lateral view, showing string-of-beads sign in the external carotid artery and complete occlusion of the internal carotid artery at a point near the bifurcation of the common carotid artery. center: Right carotid angiogram, right oblique anteroposterior view, showing an anterior communicating artery aneurysm (arrow). Blood is supplied to the contralateral side via the anterior communicating artery. right: Left vertebral angiogram, anteroposterior view, showing a fusiform aneurysm in the left vertebral artery and blood supply to the left middle cerebral artery region via the posterior communicating artery. 
right). The size of the aneurysm had not changed. The territory of the left middle cerebral artery was supplied via the anterior communicating artery from the right internal carotid artery and via the posterior communicating artery from the basilar artery.

Two days after admission, the neck of the anterior communicating artery aneurysm was clipped using a No. 3 Sugita clip through a right trans-Sylvian approach. The surface of the $A_{1}$ portions of the bilateral anterior cerebral arteries and the dome of aneurysm demonstrated no macroscopic abnormalities. However, the intracranial portion of the left internal carotid artery had become hard and white.

Postoperatively, she developed meningitis, which responded well to the intravenous administration of antibiotics. Otherwise, her postoperative course was uneventful, and she was discharged without new neurological deficits on August 31. Follow-up single photon emission computed tomography showed no hypoperfusion in any area of the brain.

\section{Discussion}

Cervicocephalic FMD cases rarely demonstrate changes in the main arteries, with only four such cases reported. ${ }^{1,5,14,24)}$ Only one patient presented with de novo formation of an aneurysm in the pericallosal artery based on angiographic evidence obtained at an interval of 9 years. ${ }^{\text {.) }}$ Our patient had developed two new lesions in the 10 years since the first admission, complete occlusion of the left internal carotid artery and a new saccular aneurysm of the anterior communicating artery.

The pathogenesis of intracranial saccular aneurysms involves two particularly important factors. The first is the presence of congenital developmental anomalies of the arterial wall. ${ }^{2,16)}$ Intrinsic abnormalities in the wall of cerebral arteries may favor the formation and rupture of cerebral aneurysms. ${ }^{3)}$ Congenital factor(s) may be more important in aneurysms arising in childhood, because these present different clinical aspects to aneurysms in adults. ${ }^{15)}$ Our patient demonstrated macroscopic disorders of the arterial wall due to the underlying FMD, although in another location to the aneurysms. The second factor is the progressive alteration of the cerebral arteries due to the mechanical action of blood flow or to arteriosclerosis due to acquired factors. ${ }^{17)}$ In moyamoya disease, cerebral aneurysms frequently occur in the vertebrobasilar system. ${ }^{23)}$ Saccular aneurysms are sometimes seen in the main feeders of arteriovenous malformations. ${ }^{21)}$ Matsuda et al. ${ }^{11)}$ reported seven cases of ruptured cerebral aneurysms arising in the collateral arteries in patients with occluded main cerebral arteries. The de novo formation of aneurysms following carotid ligation indicates the importance of hemodynamic stress in cerebral aneurysm formation. ${ }^{4,17}$ Cerebral aneurysms could be induced experimentally in hypertensive, $\beta$ aminopropionitrile-treated rats and monkeys with the carotid artery ligated unilaterally or bilaterally. ${ }^{89}$ ) In our patient, hemodynamic stress due to the internal carotid artery occlusion was probably involved in the de novo formation of the aneurysm in the anterior communicating artery.

The natural course of cervicocephalic FMD is considered to be relatively benign, and some authors warn against surgical intervention. ${ }^{20,22)}$ However, in our patient the SAH necessitated clipping of the anterior communicating artery aneurysm associated with cervicocephalic FMD.

\section{References}

1) Abdul-Rahman AM, Abu-Salih, Brun $\mathrm{A}$, Kin $\mathrm{H}$, Ljunggren B, Mizukami M, Moquist-Olsson I, Sahlin Ch, Svendgaard NAa, Thulin C-A: Fibromuscular dysplasia of the cervico-cephalic arteries. Surg Neurol 9: 217-222, 1978

2) Bremer JL: Congenital aneurysms of the cerebral arteries. An embryologic study. Arch Path (Chicago) 35: 819-831, 1943

3) Chyatte D, Reilly J, Tilson MD: Morphometric analysis of reticular and elastin fibers in the cerebral arteries of patients with intracranial aneurysms. Neurosurgery 26: 939-943, 1990

4) Dyste GN, Beck DW: De novo aneurysm formation following carotid ligation: Case report and review of the literature. Neurosurgery 24: 88-92, 1989

5) Galligioni F, Iraci G, Marin G: Fibromuscular hyperplasia of the extracranial internal carotid artery. $J$ Neurosurg 34: 647-651, 1971

6) George B, Mourier KL, Gelbert F, Raizine D, Raggueneau JL: Vascular abnormalities in the neck associated with intracranial aneurysms. Neurosurgery 24: 499-508, 1989

7) Harrington $\mathrm{OB}$, Crosby VG, Nicholas L: Fibromuscular hyperplasia of the internal carotid artery. Ann Thorac Surg 9: 516-524, 1970

8) Hashimoto N, Handa H, Hazama F: Experimentally induced cerebral aneurysms in rats: Part V. Relation of hemodynamics in the circle of Willis to formation of aneurysms. Surg Neurol 13: 41-45, 1980

9) Hashimoto N, Kim C, Kikuchi H, Kojima M, Kang Y, Hazama F: Experimental induction of cerebral aneurysms in monkeys. $J$ Neurosurg 67: 903-905, 1987 
10) Itoyama $Y$, Fukumura $A$, Nonaka $N$, Itoh $Y$, Takamura S, Matsukado Y: Fibromuscular dysplasia accompanied by giant intracranial fusiform aneurysm. Report of two cases. Neurol Med Chir (Tokyo) 28: 579-583, 1988

11) Matsuda M, Handa J, Saito A, Matsuda I, Kamijo Y: Ruptured cerebral aneurysms associated with arterial occlusion. Surg Neurol 20: 4-12, 1983

12) Mettinger KL: Fibromuscular dysplasia and the brain. II: Current concept of the disease. Stroke 13: 53-58, 1982

13) Mettinger KL, Ericson K: Fibromuscular dysplasia and the brain. Observations on angiographic, clinical and genetic characteristics. Stroke 13: 46-52, 1982

14) Osborn AG, Anderson RE: Angiographic spectrum of cervical and intracranial fibromuscular dysplasia. Stroke 8: 617-626, 1977

15) Ostergaard JR: Aetiology of intracranial saccular aneurysms in childhood. Br J Neurosurg 5: 575-580, 1991

16) Padget DH: The circle of Willis: Its embryology and anatomy, in Dandy WE (ed): Intracranial Arterial Aneurysms. Ithaca, Comstock Publishing, 1944, pp $67-90$

17) Salar G, Mingrino S: Development of intracranial saccular aneurysms: Report of two cases. Neurosurgery 8: 462-465, 1981

18) So EL, Toole JF, Dalal P, Moody DM: Cephalic fibromuscular dysplasia in 32 patients. Clinical findings and radiologic features. Arch Neurol 38:
$619-622,1981$

19) Stanley JC, Fry WJ, Seeger JF, Hoffman GL, Gabrielsen TO: Extracranial internal carotid and vertebral artery fibrodysplasia. Arch Surg 109: 215 222,1974

20) Stewart MT, Moritz MW, Smith RB III, Fulenwider JT, Perdue GD: The natural history of carotid fibromuscular dysplasia. J Vasc Surg 3: 305-310, 1986

21) Tognetti $F$, Limoni $P$, Testa $C$ : Aneurysm growth and hemodynamic stress. Surg Neurol 20: 74-78, 1983

22) Well RP, Smith RR: Fibromuscular dysplasia of the internal carotid artery: A long term follow-up. Neurosurgery 10: 39-43, 1982

23) Yabumoto $M$, Funahashi $K$, Fujii $T$, Hayashi $S$, Komai N: Moyamoya disease associated with intracranial aneurysm. Surg Neurol 20: 20-24, 1983

24) Yamamoto I, Kageyama $N$, Usui $K$, Yoshida J: Fibromuscular dysplasia of the internal carotid artery. Unusual angiographic changes with progression of clinical symptoms. Acta Neurochir (Wien) 50: 293-298, 1979

Address reprint requests to: Y. Itoyama, M.D., Section of Neurosurgery, Saiseikai Kumamoto Hospital, 4-38 Daniyama, Kumamoto 860, Japan. 